\title{
ARTÍCULO DE REVISIÓN
}

\section{Asociación de las alteraciones del sueño con el déficit de atención e hiperactividad en pediatría}

\author{
Nataly Judith Sánchez Solano ${ }^{1}$, Karen Solanyi Sarmiento Acuña², \\ Maricela Stefanía Riascos Rosero 3 , Olga Patricia Panqueva Centanaro ${ }^{4}$, \\ Álvaro Ruiz Morales ${ }^{5}$
}

\begin{abstract}
Resumen
El déficit de atención e hiperactividad (TDAH) es uno de los trastornos del comportamiento más frecuentes en pediatría, que genera conductas maladaptativas inconsistentes con la edad y el desarrollo del niño. Recientemente ha despertado gran interés la alta coexistencia de este con trastornos de sueño, al igual que una posible causa bidireccional entre las alteraciones de la arquitectura del sueño y los síntomas principales del TDAH. El objetivo de esta revisión es encontrar la asociación entre el TDAH y los trastornos del sueño en la población pediátrica, evaluando a profundidad los documentos, con el fin de encontrar nivel de evidencia, para ampliar a futuro la intervención sobre el TDAH y optimizar la calidad de vida de estos pacientes, a corto y largo plazos. Se realizó la búsqueda en Pubmed, Cochrane y Fabumed, escogiendo estudios en niños, con menos de 10 años de publicación, en inglés o español, con acceso completo. Se escogieron 52 estudios basándose en su calidad y nivel de evidencia.
\end{abstract}

Palabras clave: síndrome de apnea obstructiva del sueño, trastorno por déficit de atención con hiperactividad, trastornos del sueño.

1 Estudiante de noveno semestre de Medicina, Pontificia Universidad Javeriana, Bogotá, Colombia.

2 Estudiante de sexto semestre de Medicina, Pontificia Universidad Javeriana, Bogotá, Colombia.

3 Estudiante de décimo semestre de Medicina, Pontificia Universidad Javeriana, Bogotá, Colombia.

4 Médica pediatra neumóloga. Profesora asociada, Pontificia Universidad Javeriana-Hospital Universitario San Ignacio, Bogotá, Colombia.

5 Médico internista epidemiólogo. Profesor asociado, Pontificia Universidad Javeriana-Hospital Universitario San Ignacio, Bogotá, Colombia.

Recibido: 02/10/2013

Revisado: 09/12/2013

Aprobado: 21/01/2014 
Title: Association between Sleep Disorders and Attention Deficit and Hyperactivity in Pediatrics

\begin{abstract}
Attention deficit and hyperactive disorder (ADHD) is one of the most frequent behavioral disorders seen in the pediatric population that generates maladaptive conducts, which are inconsistent with the age and development of the child. The high coexistence between ADHD and sleep disorders, as well as the possible bidirectional cause between architectural alterations of sleep and the main symptoms of ADHD, have sparked a great interest as a topic of research. In order to find a better level of evidence, and to expand the future of interventions for ADHD to be able to optimize the quality of life in the long term for the patients, these new associations were revised more in depth in this document. The search was performed in Pubmed, Cochrane, and Fabumed, choosing studies on children, with less than ten years of publication, language in English or Spanish, and free access to the article. Fifty-two articles were chosen based on their quality and level of evidence.
\end{abstract}

Key words: Obstructive sleep apnea, attention deficit disorder with hyperactivity, sleep disorders.

\section{Introducción}

El déficit de atención e hiperactividad (TDAH) es uno de los trastornos del comportamiento más frecuentes en pediatría. Se caracteriza por la incapacidad de manejar la atención, el nivel de actividad y la impulsividad, lo que genera conductas maladaptativas inconsistentes con la edad y el desarrollo del niño. El Manual diagnóstico y estadístico de los trastornos mentales (DSM-IV) define tres tipos: predominantemente desatento
(TDAH-I), predominantemente hiperactivo-impulsivo (TDAH-HI) y combinado (TDAH-C). Básicamente, su diagnóstico es clínico, y hasta la fecha no se ha desarrollado ningún marcador biológico.

Los síntomas principales son: desatención, hiperactividad e impulsividad, y el predominio de uno de dichos síntomas establecerá el subtipo. El TDAH es un trastorno crónico, de alta comorbilidad y de causa multifactorial. Intervienen alteraciones funcionales del sistema nervioso central, lesiones anatómicas, causas genéticas y respuestas evolutivas maladaptativas [1-6].

Últimamente, se ha visto su asociación con los trastornos del sueño, lo cual genera gran interés en la población científica, pues se ha visto una posible causalidad bidireccional entre las alteraciones de la arquitectura del sueño y la aparición de los síntomas principales del TDAH. Estas nuevas asociaciones deben revisarse profundamente para encontrar mejores niveles de evidencia y, posteriormente, ampliar los niveles de intervención sobre el TDAH, para mejorar la calidad de vida de estos pacientes, a corto y largo plazos [2].

\section{Historia}

En los últimos 200 años, se ha visto la metamorfosis del TDAH, tanto en su definición como en su etiología. Quizás el primer caso reportado de TDAH en 
la literatura fue en 1798, por el médico escocés sir Alexander Crichton, en su libro titulado Una investigación sobre la naturaleza y el origen del trastorno mental: la comprensión de un sistema conciso de la fisiología y patología de la mente humana y una historia de las pasiones y sus efectos, donde se encuentra una breve descripción de casos con niños con problemas en la atención, los cuales podrían corresponder al TDAH con predominio de déficit de atención (TDAH-IA) [7]. Aproximadamente 34 años después aparece el reconocido libro Fausto, del poeta alemán Wolfgang von Goethe. Algunos autores proponen que el personaje Euforión (hijo del Dr. Fausto y Helena de Troya), que aparece en el acto 3, es un niño con TDAH de tipo combinado (TDAH-C). La siguiente aparición del trastorno en la literatura fue en 1845 , en el libro de literatura infantil Pedro Melenas, donde se describen dos casos de TDAH, en dos cuentos distintos; sin embargo, este es un tema de controversia, ya que no se puede concluir con certeza que Hoffman estuviera describiendo casos con TDAH o simplemente escribiendo cuentos para enseñarles valores morales a los niños de la época.

Las "Conferencias goulstonianas", de sir George Frederic Still, publicadas en 1902 en la revista Lancet, son consideradas "el comienzo científico de la historia del TDAH”. Él describió una serie de casos de niños con comportamiento pre- dominantemente impulsivo que tenían trastornos de conducta genéticamente determinados y que hoy día recibirían el diagnóstico de TDAH. Still relacionaba estos problemas con el retraso mental y con un déficit que él llamaba control moral [1,7-9].

Posteriormente, la mayor parte de los nombres dados al TDAH durante el siglo XX ilustra el cambio de presunciones sobre su posible etiología [10]. En 1908, Tredgold propuso la teoría de que el daño cerebral podría tener consecuencias en el comportamiento y el aprendizaje, lo cual se confirmó tras la epidemia de encefalitis letárgica que ocurrió entre 1917 y 1918, cuando muchos niños afectados comenzaron a mostrar comportamientos de "hiperquinesis, desatención, irritabilidad y destructibilidad" posterior a la infección. Como consecuencia de dicha epidemia y sus efectos deletéreos sobre el comportamiento observado en niños infectados, se acuñó el término síndrome postencefalítico, que corresponde al hoy conocido TDAH $[7,10]$.

En 1932, cambió de nombre a trastorno a enfermedad hipercinética de la infancia, gracias a la publicación de Franz Kramer y Hans Pollnow, en la cual describieron los tres síntomas principales del TDAH: hiperactividad, desatención e impulsividad. En la década de los treinta se realizaron múltiples investigaciones sobre las teorías propuestas por Tredgold, lo cual llevó a la idea de 
que el daño cerebral produce un comportamiento hiperactivo que reemplaza el síndrome postencefalítico con daño cerebral mínimo [7].

Durante esta misma década, Charles Bradley encontró tratamientos para varios trastornos comportamentales, sobre todo los relacionados con la hiperactividad, con el uso del medicamento estimulante bencedrina. En 1944, Leandro Panizzon fabricó y comercializó el metilfenidato (ritalina), una piperazina similar a la anfetamina. Ello fue un momento histórico para el TDAH, ya que este medicamento - hasta el día de hoy — es el "psicoestimulante más eficaz y el más frecuentemente formulado para el tratamiento del TDAH" [7].

En los años cuarenta y cincuenta los trabajos de Strauss y Kephart hicieron hincapié en la existencia de una lesión cerebral que explicara la existencia de los síntomas. En 1957, Laufer y colaboradores propusieron que en estos niños había una disfunción en el diencéfalo y no un daño cerebral per se.

Posteriormente, en la Conferencia de Estudio Internacional de Oxford en Neurología Infantil, en 1963, se reemplazó la expresión daño cerebral mínimo por disfunción cerebral mínima y se describieron como síntomas principales: inatención, impulsividad e hiperactividad [7,11].

En 1980, el DSM-III le cambió el nombre a trastorno de déficit de atención y se instauraron dos subtipos: con hiperactividad y sin ella y se desarrollaron tres diferentes listas de sintomatología, cada una correspondiendo a uno de los tres síntomas: desatención, impulsividad e hiperactividad [7,10].

El nombre actual se estableció con el DSM-III-R, publicado en 1987, en el cual, adicionalmente, se removieron los dos subtipos establecidos y se combinó la sintomatología de hiperactividad e impulsividad en una sola lista.

Durante la década de los noventa, los estudios encontraron en su etiología asociación genética, alteración estructural prefrontal-estriatal y cronicidad de los pacientes. También se encontraron las mismas características en los pacientes adultos.

Todo lo anterior llevó a que en 1994, con el DSM-IV, se instauraran los tres subtipos de TDAH conocidos hasta el día de hoy: combinado (TDAH-C), con predominio del déficit de atención (TDAH-IA) y con predominio hiperactivo-impulsivo (TDAH-HI). Además, se describió el TDAH en el adulto $[7,10]$.

En los últimos treinta años ha mejorado mucho la tipificación del TDAH con la identificación de los síntomas nucleares, el uso de criterios diagnósticos operativos, la detección de un componente etiológico genético y la existencia de tratamientos farmacológicos eficaces. 
Hoy día caben pocas dudas acerca de su existencia como trastorno, gracias al alto impacto generado por el deterioro del funcionamiento académico, laboral y social [12].

\section{Epidemiología}

El Centro para el Control y Prevención de Enfermedades, de Estados Unidos, considera que el TDAH representa, para la sociedad en general, un gran problema de salud pública, debido a su alta prevalencia, el deterioro en el rendimiento escolar, la cronicidad de la enfermedad y la limitada eficacia de los tratamientos. Tiene una prevalencia en Colombia de aproximadamente 3-7,5\% de los niños en edad escolar, y predomina en el sexo masculino con una relación de 3:1 $[1,2,4,10]$.

Como se mencionó, el TDAH es un trastorno crónico, que inicia en la niñez y que persiste entre el $50 \%$ y el $80 \%$ en la adultez $[1,5,10,13]$. Además, se observa un alta comorbilidad con múltiples condiciones como: déficit de coordinación motora y de balance, trastornos del lenguaje, dificultades de aprendizaje, trastornos del estado del ánimo, trastornos de ansiedad, obesidad, trastornos del sueño, abuso de sustancias, delincuencia, agarofobia y fobia social.

Adicionalmente, en los jóvenes diagnosticados antes de los 7 años de edad con TDAH se reportan problemas en funciones ejecutivas como la inhibición, la memoria de trabajo, la planificación y la atención sostenida. Este trastorno coexiste con comorbilidades entre un $59 \%$ y $87 \%$ con una única condición, y en un $20 \%$ con tres o más condiciones. También se ha notado que muchos de sus síntomas se solapan con síntomas de otros trastornos, lo cual ha hecho surgir muchas dudas en cuanto a si las condiciones coexistentes hacen parte del TDAH o si son entidades separadas $[1,2,4,5,10,13]$.

\section{Subtipos}

Según el tipo y la predominancia de síntomas presentados, el TDAH se divide en tres distintos subtipos, ya mencionados, que presentan en común manifestaciones clínicas antes de los 7 años de edad. Los síntomas se clasifican en dos categorías: síntomas de desatención y síntomas de hiperactividad-impulsividad.

La primer categoría incluye síntomas como hipoprosexia, incapacidad para organizar actividades, fallas en la memoria, dificultad para seguir instrucciones y evitar actividades que requieran esfuerzo mental. La segunda categoría abarca las siguientes características: inquietud motora, hipercinesia, locuacidad, impaciencia, intrusión y ruido. En el DSM-IV se encuentran los criterios diagnósticos para cada subtipo de TDAH y los síntomas puntuales de cada categoría $[1,3]$. 
El subtipo TDAH-C es el más diagnosticado, con una mayor frecuencia entre los 7 y los 10 años de edad, cuando alcanza una prevalencia del $45 \%$ y una presentación 4 veces mayor en el sexo masculino $[1,10]$. Este subtipo presenta una combinación de los síntomas de desatención, hiperactividad e impulsividad. Para su diagnóstico se requieren mínimo de seis síntomas mencionados y una duración de, por lo menos, 6 meses $[10,13]$.

En cambio, en el TDAH-IA están presentes 6 o más síntomas de desatención, pero menos de 6 de hiperactividad e impulsividad y una duración de, por lo menos, 6 meses. Este subtipo es mucho más frecuente en adolescentes con edades de 10 años o más y en el sexo femenino $[1,10,13]$.

El TDAH-HI es el subtipo de menor prevalencia $[3,10,14]$ y constituye alrededor del $5 \%$ de los casos, razón por la cual carece de estudios suficientes. De los 9 síntomas de la categoría de hiperactividad-impulsividad, se requiere la presencia de al menos 6 , mínimo durante 6 meses y menos de 6 síntomas de desatención, para poder realizar el diagnóstico $[1,3,10,14]$.

Ramelli y colaboradores reportan una prevalencia del 50\% de TDAH-HI en niños menores de 6 años de edad. Por ende, este subtipo es mucho más agobiante para el paciente y su familia, pues el diagnóstico y el tratamiento se da a una edad más temprana $[10,13,14]$. Adicionalmente, algunos estudios reportan que los síntomas de hiperactividad tienen una fuerte asociación con los trastornos respiratorios del sueño y que en niños con el subtipo TDAH-HI hay mayor asociación con el ronquido nocturno primario. Sin embargo, otros investigadores no han encontrado relación entre los subtipos TDAH y los trastornos del sueño $[2,5,15]$.

En cuanto a su pronóstico, realmente hay mucha controversia, probablemente debido a la falta de evidencia. Baeyens y colaboradores [10] mencionan que el TDAH-HI tiene consecuencias negativas a largo plazo; sin embargo, Escobar y colaboradores [16] encontraron que el subtipo TDAH-HI se asocia con mejor calidad de vida, menos sintomatología del trastorno y menor comorbilidad con trastornos psiquiátricos, comparado con los subtipos TDAH-C y TDAH-IA. Con los anteriores datos, es posible concluir respecto a la necesidad de obtener más evidencia, para conocer el verdadero curso de esta patología.

\section{Síndrome de apnea obstructiva del sueño}

La primera descripción conocida de este trastorno respiratorio se remonta a 1836 , cuando Charles Dickens, en su libro Los papeles póstumos del club de Pickwick, describe a Joe, un niño obeso, somnoliento en el día y que roncaba al dormir. 
Broadbent describió las características clínicas en 1877 y Well describió cómo pudo tratar este trastorno en niños corrigiendo defectos obstructivos de la vía aérea superior. En 1956, Burwell adoptó el término pickwickian, que se refiere a niños obesos, somnolientos e hipercápnicos. Y en 1965, Gastaut relacionó el síndrome con sus hallazgos polisomnográficos [17-21].

El mecanismo de obstrucción que lleva al desarrollo de apnea se debe a la relajación muscular que aparece durante el sueño, pues los músculos no pueden mantener su tono ante la presión negativa que se genera en la inspiración. El paciente puede tener las vía aérea estrecha; sin embargo, esta no causa sintomatología alguna durante el día, pero durante el sueño el tono muscular decae y la vía aérea se estrecha aún más (lo que produce oclusión y apnea). Cualquier alteración de la función faríngea que aumente la resistencia al flujo, así como factores neurológicos que favorezcan la dilatación de los músculos, puede causar una disminución de la permeabilidad de la vía aérea y ocasionar este trastorno respiratorio [22].

Los factores predisponentes del síndrome de apnea obstructiva del sueño (SAHOS) más importantes en los niños son: hipertrofia de amígdalas y adenoides (sin que ello signifique que todo niño con amígdalas grandes va a desarrollar apnea del sueño), enferme- dades neurológicas y neuromusculares que favorezcan la pérdida de tono de la musculatura faríngea (malformaciones congénitas craneofaciales, sobre todo las que involucren acortamiento del cuello, retrognatia, paladar duro elevado, barbilla pequeña y triangular como en el síndrome de Down, síndrome de Pierre Robin), entre otros. Además, se observan factores predisponentes como la obstrucción nasal marcada y la obesidad.

Los niños con trastornos de resistencia al flujo aéreo pueden presentar un amplio rango de sintomatología y manifestaciones clínicas, desde casos leves que solo involucran el ronquido al dormir, hasta episodios graves de síndrome de apnea obstructiva del sueño. El signo principal de este síndrome es el ronquido, que puede clasificarse en primario cuando no se acompaña de alteraciones polisomnográficas y en secundario si las presenta. Otros signos que indican mayor dificultad respiratoria son los movimientos paradójicos de la caja torácica, sudoración profusa secundaria al esfuerzo ventilatorio y posturas de sueño anormales como la hiperextensión del cuello para mejorar la entrada de aire.

Algunos niños pueden cursar con náuseas y vómito debido al aumento de la presión negativa intratorácica, que progresa incluso a reflujo gastroesofágico. Además, se encuentra asociación con algunos trastornos del sueño, como terror nocturno, pesadillas, sonambulis- 
mo, bruxismo, enuresis e hipersomnolencia, en algunos casos [23].

\section{TDAH y trastornos del sueño}

Durante los últimos años se ha visto que la privación y la interrupción del sueño generan síntomas semejantes a los observados en el TDAH. Esta conexión ha estimulado series de investigaciones para encontrar un vínculo entre el TDAH y los trastornos del sueño, y se ha encontrado una asociación hasta del $50 \%$ entre ellas y un aumento de hasta cinco veces más la probabilidad de reportar problemas en el sueño. Asimismo, también se ha visto una alta tasa de reportes de problemas en el sueño, de padres con niños con TDAH [24-30].

Gracias a los estudios también se han logrado identificar otros factores relacionados con los trastornos del sueño. Dos recientes metanálisis demostraron que la edad, el sexo, las mediciones del sueño, las comorbilidades, los trastornos psiquiátricos y el uso de medicación estimulante presentan una correlación directa, entre las características del sueño y el síndrome por déficit de atención.

En el 2009, Li y colaboradores [28] publicaron el estudio multicéntrico más grande de TDAH que se ha llevado a cabo en China, donde se encontró que el sexo masculino, un bajo nivel de educación, bajos ingresos económicos de los padres, familias monoparenterales e historia previa de diagnóstico de TDAH tienen una mayor asociación con trastornos del sueño [28,31,32].

Adicionalmente, los estudios han permitido determinar la importancia de los neurotransmisores noradrenérgicos y dopaminérgicos en las alteraciones del sueño, asociados al déficit de atención; sin embargo, los resultados no han sido concluyentes. Existen hipótesis sobre el origen de los trastornos del sueño en los niños con TDAH: se han descrito modelos de comportamiento, ciclos circadianos y factores genéticos, para explicar los mecanismos asociados con la interrupción del sueño y con los diversos problemas de comportamiento que presentan $[4,8-10,33]$.

Recientemente, ha surgido una nueva hipótesis que correlaciona el TDAH y el estado nutricional del individuo, ya que se ha visto su asociación con mal nutrición, dada principalmente por déficit de ácidos grasos esenciales y hierro. Por lo tanto, es importante en la evaluación del paciente con TDAH el estudio de su estado nutricional, por ser este un factor corregible que permite mejorar la condición neurocognitiva y comportamental del niño [34,35]. Yehuda y colaboradores [34] presentaron en un estudio cómo la calidad de vida de los niños aumentó, al igual que hubo un mejoramiento en la calidad del sueño, mayor capacidad de concentración y menor fatiga diurna después de la administración de suple- 
mentos de hierro y ácidos grasos esenciales en relación con el grupo tratado con placebo [35].

Los estudios realizados hasta la fecha han logrado demostrar que existe una alteración en la arquitectura del sueño en los niños con TDAH [36-41], lo que podría generar las alteraciones del sueño observadas en esta población, dentro de las cuales se encuentran: insomnio de conciliación, disminución de necesidad del sueño, aumento en la latencia de sueño (o en tiempo para quedarse dormido), sueño inquieto, despertares nocturnos, dificultad para despertar en las mañanas, hipersomnia y fatiga diurna, enuresis, ronquidos, síndrome de apnea obstructiva del sueño, trastornos de la respiración durante el sueño (SDB), síndrome de piernas inquietas (RLS) y síndrome de movimientos periódicos de las piernas (PLMD).

Dentro de las alteraciones en la arquitectura se encuentran: aumento de la latencia en fase REM o fase de movimientos oculares rápidos del sueño, disminución del porcentaje de sueño REM, aumento del porcentaje de sueño de ondas lentas (SWS) y disminución del patrón alternante cíclico, lo cual sugiere un estado de hipoalertamiento e inestabilidad del sueño. Adicionalmente, también se ha visto una relación entre trastornos del sueño y dificultades de aprendizaje, característica que comparte con el TDAH [2,24]. Por lo anterior, se sospecha que puede existir una vía neu- robiológica compartida entre estas dos entidades [2,24,26-29,33,36-41].

Hvolby y colaboradores [24] demostraron en su estudio que el $49 \%$ de los niños con TDAH presentaron dificultades al dormir, dentro de las cuales los más prevalentes fueron los problemas en la iniciación de sueño y el sueño inquieto. Ellos plantearon que estas perturbaciones del sueño deben ser "vistas como una complicación en niños con TDAH”.

Adicionalmente, propusieron que el mejor diseño para estudiar la asociación necesita la inclusión de cuestionarios y notas diarias, ya que de esta manera se obtiene un mejor registro del patrón del sueño [24]. A pesar de ser el único estudio que ha utilizado cámara infrarroja para filmar la polisomnografía, no se encontraron diferencias estadísticamente significativas entre las variables del sueño medidas, en niños con TDAH y los controles; pero se logró demostrar que en el TDAH existe un aumento del número de movimientos de las cuatro extremidades y que la duración de estos es el doble comparado con los controles. Adicionalmente, se concluye que medir los movimientos con electroencefalograma y polisomnograma contribuye a establecer los marcadores de gravedad del trastorno [41-43].

Adicionalmente, se encontró que la actividad nocturna es directamente proporcional a la agitación diurna: se vio 
implicada la dopamina en el circuito fisiopatológico del TDAH y los trastornos del sueño, por lo cual Konofal y colaboradores [29] concluyeron que "el TDAH no es un trastorno puramente diurno, sino que también tiene repercusiones en el sueño".

De la misma forma, se han descrito diferencias en la arquitectura del sueño dentro de los mismos subtipos del TDAH. Un estudio publicado en el 2009 demostró que existe una prevalencia de patologías del sueño en los padres de los pacientes pediátricos con TDAH del subtipo TDAH-C, pero no en niños con TDAH-HI.

Por ende, los problemas del sueño más comunes en TDAH (dificultad para quedarse dormido, despertares nocturnos, parasomnias, menos duración del sueño, inquietud durante el sueño) se presentan únicamente en el TDAH-C. Caso contrario ocurre en el TDAH-HI, el cual está relacionado con una mayor hipersomnia diurna comparado con el TDAH-C y el grupo control [30,41].

En la evaluación del niño con TDAH es necesario el estudio de la higiene del sueño y el ambiente en el que se desarrolla el inicio del sueño [44]. Además, esta evaluación del sueño también serviría como una herramienta de tamizaje para encontrar presencia de trastornos del sueño, dada su alta prevalencia en esta enfermedad [32]. De esta manera, se podría dar un manejo temprano y reducir los síntomas comportamentales, al igual que el uso de medicamentos psicotrópi$\cos$ [45]. Según un estudio realizado por Rodopman y colaboradores [44], existe la posibilidad de plantear la realización de un perfil del sueño, cuyo objetivo sea lograr una mejor higiene del sueño con la consiguiente disminución de sintomatología y aumento de rendimiento diurno basado en el logro de adecuados hábitos de sueño en cooperación entre niños y padres.

Es importante hacer hincapié en que los estudios realizados han tenido limitaciones metodológicas, dificultades para establecer muestras poblacionales adecuadas y ausencia de instrumentos estandarizados para la evaluación del comportamiento nocturno. Por consiguiente, las investigaciones anteriores tienen diferentes diseños experimentales, que utilizan desde cuestionarios a padres, profesores y pacientes; realización de diarios para registrar patrones de sueño por parte de los sujetos; medición de trastornos de sueño con polisomnografía, actigrafía, electroencefalograma, filmación de video o combinación de estas variables, y diferentes métodos de selección de muestra.

Consecuentemente, esto ha creado limitaciones a la hora de analizar y realizar conclusiones, ya que no solamente por las distintas metodologías los estudios son incomparables, sino que además 
existen muchas variables de confusión y discrepancias en los resultados. Por ello es importante resaltar la necesidad de realización de más estudios, que minimicen estas distintas variables para lograr unificar la evidencia que existe hasta la fecha $[2,24,32,45]$.

\section{TDAH y síndrome de movimientos periódicos de las piernas}

Como se mencionó, dentro de las parasomnias que se encuentran frecuentemente en niños con TDAH, el PLMD constituye el de más fuerte asociación. En 1999, Picchietti y Walters señalaron que el $91 \%$ de niños con TDAH presentaba PLMD [42]. Posteriormente, Crabtree y colaboradores, además de encontrar que el $63 \%$ de niños con TDAH roncaba al dormir, señalaron que el $26 \%$ cursaba también con trastornos del ánimo y ansiedad, y el $36 \%$, con PLMD [27]. Similarmente, el estudio chino de Li y colaboradores demostró una asociación sólida entre TDAH y PLMD $[2,27,28,37,39,42]$. En contraste, en el 2001, Chervin y Hedger demostraron que no existía relación entre TDAH y PLMD.

Después el estudio de O'Brien y colaboradores, del 2003, incorporó niños con TDAH "referidos" a centros de sueño y niños con TDAH obtenidos por medios de encuestas en colegios, es decir, de la comunidad. Así se evidenciaron diferencias entre estos dos grupos: la población con TDAH obtenida de la comunidad representó la mayoría de los casos de TDAH y en este estudio no se encontró coexistencia con PLMD, en contraste con la evidencia previa. Sin embargo, en la población referida, a la cual llamaron TDAH clínico, se observó una alta prevalencia de PLMD. Consecuentemente, se relacionó el grupo de pacientes con TDAH y PLMD con la mayor sintomatología en la disrupción del sueño. Finalmente, Sadeh y colaboradores [32], en el 2006, demostraron un aumento de la prevalencia de PLMD en individuos con TDAH, que fue el único trastorno de sueño que se encontró presente en esta población. Estos hallazgos corroboran la necesidad de realizar más estudios para aclarar esta asociación descrita $[26,43]$.

\section{TDAH y psicoestimulantes}

Otra de las polémicas dentro de esta área de estudio es la influencia de los fármacos psicoestimulantes, específicamente el metilfenidato y las anfetaminas, en los trastornos del sueño, debido a que la evidencia así lo ha demostrado. Esto es de gran importancia, por las implicaciones que acarrearía la prevalencia de trastornos del sueño en TDAH, secundario a su tratamiento, ya que estos fármacos pueden causar aumento de la latencia y disminución de la duración del sueño, además de disminuir su calidad [46]. En un estudio de cohorte se logró comprobar que "la terapia psicoestimulante no 
tiene ningún efecto en las características del sueño" [37]. Mayes y colaboradores, en el 2009, también demostraron que no hubo diferencia estadísticamente significativa entre el grupo medicado y el no medicado, después de realizar un análisis covariado. Sin embargo, se requiere más evidencia para poder afirmarlo, especialmente por medio de un estudio clínico controlado con placebo y psicoestimulantes [30,37].

\section{TDAH y síndrome de apnea obstructiva del sueño}

El SAHOS puede definirse como un trastorno respiratorio que se desarrolla durante el sueño y que consiste en periodos de apnea con obstrucción completa e intermitente al flujo respiratorio o hipopnea, en el que hay una obstrucción parcial; pero prolongada de la vía aérea, los eventos de apnea se preceden de ronquido fuerte, la persona despierta y los síntomas ceden; posteriormente, debido a la interrupción del tiempo de sueño, se genera una excesiva somnolencia diurna [22].

Se ha observado que niños con SAHOS presentan síntomas de hiperactividad e inatención en un $95 \%$, muy frecuentemente asociados al TDAH $[38,47]$. También se ha demostrado mejoría de los síntomas posterior al tratamiento con adenotonsilectomía (AT), presión positiva continua de la vía aérea (CPAP) o presión positiva binivelada de la vía aérea (BiPAP). Se ha observado una asociación importante entre el TDAH, los niños roncadores y la respiración alterada durante el sueño (SDB, por su sigla en inglés), un tipo de evento obstructivo donde hay impedimento periódico de la respiración durante el sueño.

Se ha descrito que hasta un tercio de los pacientes que presentan ambas entidades poseen, además, un comportamiento impulsivo e hiperactivo. El SDB se ha convertido en la entidad predecesora al SAHOS, debido a que solo necesitaría de la hipersomnia diurna para completar el criterio diagnóstico. Adicionalmente, se sabe que el SAHOS exacerba los síntomas de TDAH y plantea que existe una relación unidireccional, bidireccional o causal entre el SAHOS y el TDAH $[38,47,48]$.

La fisiopatología de esta asociación todavía es incierta; sin embargo, se expone como teoría que la apnea recurrente genera hipoxia episódica y produce daño neurocognitivo por medio de tres mecanismos: 1) Por aumento de glutamato secundario a la hipoxia, con excitotoxicidad de las neuronas hipocampales, que reducen su volumen y, por consiguiente, disminuyen la consolidación de la memoria, al igual que el volumen de las cortezas parietales y frontales. 2) Por incremento de radicales libres secundario a hipoxia, pues al aumentar la peroxidación lipídica, causa daño miocárdico, cerebral y disfunción endotelial. Y 3) Por la producción de 
óxido nítrico (vasodilatador), que lleva a un desbalance entre los vasodilatadores y vasoconstrictores, y termina en disfunción endotelial también.

Posteriormente, la disfunción endotelial aumenta la coagulabilidad, que predispone a formación de ateromas. Finalmente, la vía común es la disminución del flujo cerebral. Las funciones neurocognitivas más frecuentemente afectadas son el funcionamiento ejecutivo, el razonamiento, la atención, la vigilancia, el aprendizaje y la memoria. En niños hay más predisposición para la irritabilidad, disprosexia, hipovigilancia, inestabilidad emocional y disminución de la inteligencia $[34,35,38,47,49]$.

O'Brien y colaboradores [38] demostraron que existe una asociación entre SAHOS y TDAH, con una comorbilidad de aproximadamente el $25 \%$. Se encontró una alta prevalencia de SAHOS en la población que marca hiperactividad moderada en la escala de Conneer's. Consecuentemente, concluyen que el SAHOS puede inducir y exacerbar los síntomas hiperactivos, pero no puede imitar un TDAH severo $[35,38]$.

En un estudio publicado en el 2006 se realizó seguimiento de un año a pacientes con SDB y SAHOS postadenotonsilectomía, que presentaban síntomas de hiperactividad e impulsividad o diagnóstico de TDAH. Se demostró que la adenotonsilectomía en niños con SAHOS no solamente mejora o disminuye los síntomas de TDAH, sino que también revierte el daño neurocognitivo $[34,48,50]$. Estos hallazgos fueron similares a los obtenidos por Huang y colaboradores [51], quienes también encontraron que el tratamiento quirúrgico tiene una marcada mejoría de la sintomatología de TDAH y que, además, puede prevenir el uso crónico de metilfenidato y sus potenciales efectos adversos.

Es importante mencionar que el SAHOS se puede enmascarar como un TDAH y que el tratamiento con psicoestimulantes puede mejorar los síntomas del SAHOS y prolongar su diagnóstico. Resalta entonces la importancia de descartar trastornos en el sueño, como el SAHOS, y trastornos bioquímicos que subyacen a los déficits de función ejecutiva y atención, antes de realizar el diagnóstico de TDAH [50,52].

Con respecto a la sintomatología prevalente, la revisión sistemática de Cortese y colaboradores [31] indica que los niños con TDAH tienen una mayor somnolencia diurna, más movimientos durante el sueño y los índices de apneahipopnea son superiores en comparación con los controles.

\section{Conclusión}

En los niños con TDAH se han encontrado alteraciones en la arquitectura del sueño, que presentan una alta comorbili- 
dad con trastornos del sueño como SDB, PLMD, roncar e insomnio. El TDAH en pediatría está relacionado principalmente con SAHOS. Las dos alteraciones comparten mecanismos neurobiológicos y solapamiento entre los síntomas de ambos trastornos; por lo tanto, el tamizaje y el diagnóstico de SAHOS en pacientes con manifestaciones clínicas compatibles con TDAH permitirá lograr la oportuna intervención médica para el control de síntomas de forma temprana.

Adicionalmente, hasta la fecha hace falta evidencia de la asociación estadística y correlación del TDAH y los trastornos del sueño, secundarios a la variabilidad metodológica en los estudios. Estos hallazgos sugieren la necesidad de realización de estudios de asociación y de correlación entre las dos entidades, con adecuados muestreos, mediciones prolongadas en el tiempo, control de variables ambientales y exclusión de otras comorbilidades como trastornos del ánimo y de ansiedad.

\section{Referencias}

1. Reiff MI, Stein MT. Attention-deficit/ hyperactivity disorder evaluation and diagnosis: a practical approach in office practice. Pediatr Clin North Am. 2003 Oct;50(5):1019-48.

2. Spruyt K, Gozal D. Sleep disturbances in children with attention-deficit/hyperactivity disorder. Expert Rev Neurother. 2011 Apr;11(4):565-77.
3. Rappley MD. Clinical practice. Attention deficit-hyperactivity disorder. $\mathrm{N}$ Engl J Med. 2005 Jan 13;352(2):165-73.

4. Biederman J, Faraone SV. Attentiondeficit hyperactivity disorder. Lancet 2005;366(9481):237-48.

5. Yoon SY, Jain U, Shapiro C. Sleep in attention-deficit/hyperactivity disorder in children and adults: past, present, and future. Sleep Med Rev. 2012 Aug;16(4):371-88.

6. Wagner BJ. Attention deficit hyperactivity disorder: current concepts and underlying mechanisms. J Child Adolesc Psychiatr Nurs. 2000;13(3):113-24.

7. Lange KW, Reichl S, Lange KM, Tucha L, Tucha O. The history of attention deficit hyperactivity disorder. Atten Defic Hyperact Disord. 2010 Dec;2(4):241-55.

8. Bonazza S, Scaglione C, Poppi M, Rizzo G. Did Goethe describe attention deficit hyperactivity disorder? Eur Neurol. 2011;65(2):70-1.

9. Still G. Some abnormal psychical conditions in children: the Goulstonian lectures. Lancet. 1902;1:1008-12.

10. Baeyens D, Roeyers H, Walle JV. Subtypes of attention-deficit/hyperactivity disorder (ADHD): distinct or related disorders across measurement levels? Child Psychiatr Hum Dev. 2006 Summer;36(4):403-17.

11. Wood DR, Reimherr FW, Wender PH, Johnson GE. Diagnosis and treatment of minimal brain dysfunction in adults: a preliminary report. Arch Gen Psychiatry. 1976 Dec;33(12):1453-60.

12. Spencer T, Biederman J, Wilens TE, Faraone SV. Adults with attention-deficit/ hyperactivity disorder: a controversial diagnosis. J Clin Psychiatry. 1998;59 Suppl 7:59-68. 
13. Ramelli GP, Zanda N, Bianchetti MG, Leoni F. Age-dependent presentation in children with attention deficit hyperactivity disorder. World J Pediatr. 2010 Feb;6(1):90.

14. Ghanizadeh A. Is presentation of ADHD subtypes different between community and clinical samples? World J Pediatr. 2010 Nov;6(4):372.

15. Tsai MH, Huang YS. Attention-deficit/ hyperactivity disorder and sleep disorders in children. Med Clin North Am. 2010 May;94(3):615-32.

16. Escobar R, Hervas A, Soutullo C, Mardomingo MJ, Urunuela A, Gilaberte I. Attention deficit/hyperactivity disorder: burden of the disease according to subtypes in recently diagnosed children. Actas Esp Psiquiatr. 2008 SepOct;36(5):285-94.

17. Dickens C. The posthumous papers of the Pickwick club. London: Chapman and Hall; 1836.

18. Dement W. History of sleep physiology and medicine. In Kryger M, Roth T, Dement $\mathrm{W}$, editors. Principles of practice of sleep medicine. 3rd ed. Philadelphia: WB Saunders; 2000. p. 1-14.

19. Thorpy M. Historical perspective on sleep and man. In Culebras A, editor. Sleep disorders and neurological disease. New York: Marcel Dekker; 2000. p. 1-36.

20. Bickelmann AG, Burwell CS, Robin ED, Whaley RD. Extreme obesity associated with alveolar hypoventilation; a Pickwickian syndrome. Am J Med. 1956 Nov;21(5):811-8.

21. Gastaut H, Tassinari CA, Duron B. Polygraphic study of diurnal and nocturnal (hypnic and respiratory) episodal manifestations of Pickwick syndrome. Rev Neurol (Paris). 1965 Jun;112(6):568-79.
22. Braunwald E, Fauci A, Isselbacher K, Kasper D, Hauser S, Longo D, et al. Harrison's principles of internal medicine 18th edition. 18th ed. New York: McGraw Hill; 2012.

23. Villa J, Martínez C, Pérez Pérez G, Cortell A, Gómez-Pastrana D, Álvarez G, et al. Síndrome de apneas-hipopneas del sueño. Asociación Española de Pediatría. 2008:111-125.

24. Hvolby A, Jorgensen J, Bilenberg N. Parental rating of sleep in children with attention deficit/hyperactivity disorder. Eur Child Adolesc Psychiatry. 2009 Jul;18(7):429-38.

25. Sung V, Hiscock H, Sciberras E, Efron D. Sleep problems in children with attention-deficit/hyperactivity disorder: prevalence and the effect on the child and family. Arch Pediatr Adolesc Med. 2008 Apr;162(4):336-42.

26. O’Brien LM, Ivanenko A, Crabtree VM, Holbrook CR, Bruner JL, Klaus CJ, et al. Sleep disturbances in children with attention deficit hyperactivity disorder. Pediatr Res. 2003 Aug;54(2):237-43.

27. Crabtree VM, Ivanenko A, Gozal D. Clinical and parental assessment of sleep in children with attention-deficit/hyperactivity disorder referred to a pediatric sleep medicine center. Clin Pediatr ( $\mathrm{Ph}$ ila). 2003 Nov-Dec;42(9):807-13.

28. Li S, Jin X, Yan C, Wu S, Jiang F, Shen $X$. Sleep problems in Chinese schoolaged children with a parent-reported history of ADHD. J Atten Disord. 2009 Jul;13(1):18-26.

29. Konofal E, Lecendreux M, Bouvard MP, Mouren-Simeoni MC. High levels of nocturnal activity in children with attention-deficit hyperactivity disorder: a video analysis. Psychiatry Clin Neurosci. 2001 Apr;55(2):97-103. 
30. Mayes SD, Calhoun SL, Bixler EO, Vgontzas AN, Mahr F, Hillwig-Garcia J, et al. ADHD subtypes and comorbid anxiety, depression, and oppositional-defiant disorder: differences in sleep problems. $\mathrm{J}$ Pediatr Psychol. 2009 Apr;34(3):328-37.

31. Cortese S, Konofal E, Yateman N, Mouren MC, Lecendreux M. Sleep and alertness in children with attentiondeficit/hyperactivity disorder: a systematic review of the literature. Sleep. 2006 Apr;29(4):504-11.

32. Sadeh A, Pergamin L, Bar-Haim Y. Sleep in children with attention-deficit hyperactivity disorder: a meta-analysis of polysomnographic studies. Sleep Med Rev. 2006 Dec;10(6):381-98.

33. Corkum P, Moldofsky H, Hogg-Johnson S, Humphries T, Tannock R. Sleep problems in children with attention-deficit/hyperactivity disorder: impact of subtype, comorbidity, and stimulant medication. J Am Acad Child Adolesc Psychiatry. 1999 Oct;38(10):1285-93.

34. Yehuda S, Rabinovitz-Shenkar S, Carasso RL. Effects of essential fatty acids in iron deficient and sleep-disturbed attention deficit hyperactivity disorder (ADHD) children. Eur J Clin Nutr. 2011 Oct;65(10):1167-9.

35. Gozal D, Kheirandish-Gozal L. Iron deficiency and periodic leg movement disorder of sleep. Sleep Med. 2009 Feb;10(2):265; author reply 265-7.

36. Stein MA. Unravelling sleep problems in treated and untreated children with ADHD. J Child Adolesc Psychopharmacol. 1999;9(3):157-68.

37. O'Brien LM, Ivanenko A, Crabtree VM, Holbrook CR, Bruner JL, Klaus CJ, et al. The effect of stimulants on sleep characteristics in children with attention deficit/ hyperactivity disorder. Sleep Med. 2003 Jul;4(4):309-16.
38. O'Brien LM, Holbrook CR, Mervis CB, Klaus CJ, Bruner JL, Raffield TJ, et al. Sleep and neurobehavioral characteristics of 5- to 7-year-old children with parentally reported symptoms of attention-deficit/hyperactivity disorder. Pediatrics. 2003 Mar;111(3):554-63.

39. LeBourgeois MK, Avis K, Mixon M, Olmi J, Harsh J. Snoring, sleep quality, and sleepiness across attention-deficit/ hyperactivity disorder subtypes. Sleep. 2004 May 1;27(3):520-5.

40. Gruber R, Sadeh A, Raviv A. Instability of sleep patterns in children with attention-deficit/hyperactivity disorder. J Am Acad Child Adolesc Psychiatry. 2000 Apr;39(4):495-501.

41. Miano S, Donfrancesco R, Bruni O, Ferri R, Galiffa S, Pagani J, et al. NREM sleep instability is reduced in children with attention-deficit/hyperactivity disorder. Sleep. 2006 Jun;29(6):797-803.

42. Picchietti DL, Walters AS. Moderate to severe periodic limb movement disorder in childhood and adolescence. Sleep. 1999 May 1;22(3):297-300.

43. Chervin RD, Hedger KM. Clinical prediction of periodic leg movements during sleep in children. Sleep Med. 2001 Nov;2(6):501-10.

44. Rodopman-Arman A, Perdahli-Fis N, Ekinci O, Berkem M. Sleep habits, parasomnias and associated behaviors in school children with attention deficit hyperactivity disorder (ADHD). Turk J Pediatr. 2011 Jul-Aug;53(4):397-403.

45. Owens JA. Sleep disorders and attentiondeficit/hyperactivity disorder. Curr Psychiatry Rep. 2008 Oct;10(5):439-44.

46. Rostain AL. Sleep disturbances and ADHD medications. Curr Psychiatry Rep. 2007 Oct;9(5):399-400. 
47. Youssef NA, Ege M, Angly SS, Strauss JL, Marx CE. Is obstructive sleep apnea associated with ADHD? Ann Clin Psychiatry. 2011 Aug;23(3):213-24.

48. Chervin RD, Ruzicka DL, Giordani BJ, Weatherly RA, Dillon JE, Hodges EK, et al. Sleep-disordered breathing, behavior, and cognition in children before and after adenotonsillectomy. Pediatrics. 2006 Apr;117(4):e769-78.

49. Lal C, Strange C, Bachman D. Neurocognitive impairment in obstructive sleep apnea. Chest. 2012 Jun;141(6):1601-10.

50. Gozal D, O'Brien L, Row BW. Consequences of snoring and sleep disordered breathing in children. Pediatr Pulmonol Suppl. 2004;26:166-8.

51. Huang YS, Guilleminault C, Li HY, Yang $\mathrm{CM}, \mathrm{Wu}$ YY, Chen NH. Attention-deficit/ hyperactivity disorder with obstructive sleep apnea: a treatment outcome study. Sleep Med. 2007 Jan;8(1):18-30.

52. O'Brien LM, Gozal D. Sleep in children with attention deficit/hyperactivity disorder. Minerva Pediatr. 2004 Dec;56(6):585-601.

\section{Correspondencia}

Nataly Judith Sanchez Solano natajudi58@hotmail.com

Karen Solanyi Sarmiento Acuña kssarmiento@gmail.com

Maricela Stefanía Riascos Rosero abigar2539@hotmail.com 